\title{
Covalent surface modifications and superconductivity of two-dimensional metal carbide MXenes
}

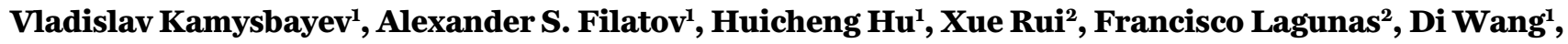 \\ Robert F. Klie ${ }^{2}$, Dmitri V. Talapin ${ }^{1,3 *}$
}

${ }^{1}$ Department of Chemistry and James Franck Institute, University of Chicago, Chicago, IL 60637, USA. '2Department of Physics, University of Illinois at Chicago, Chicago, IL 60607, USA. ${ }^{3}$ Center for Nanoscale Materials, Argonne National Laboratory, Argonne, IL 60439, USA.

*Corresponding author. Email: dvtalapin@uchicago.edu

Versatile chemical transformations of surface functional groups in 2D transition-metal carbides (MXenes) open up a new design space for this broad class of functional materials. We introduce a general strategy to install and remove surface groups by performing substitution and elimination reactions in molten inorganic salts. Successful synthesis of MXenes with $\mathrm{O}, \mathrm{NH}, \mathrm{S}, \mathrm{Cl}, \mathrm{Se}, \mathrm{Br}$, and Te surface terminations, as well as bare MXenes (no surface termination) was demonstrated. These MXenes show distinctive structural and electronic properties. For example, the surface groups control interatomic distances in the MXene lattice, and $\mathrm{Ti}_{n+1} \mathrm{C}_{n}(n=1,2)$ MXenes terminated with $\mathrm{Te}^{2-}$ ligands show a giant, $(>18 \%)$ in-plane lattice expansion compared to the bulk $\mathrm{TiC}$ lattice. $\mathrm{Nb}_{2} \mathrm{C}$ MXenes exhibited surface-group-dependent superconductivity.

Two-dimensional (2D) transition-metal carbides and nitrides (MXenes) (1) have been actively studied for applications in supercapacitors (2), batteries (3), electromagnetic interference shielding (4), composites (5, 6), and catalysts (7). MXenes are typically synthesized from the corresponding MAX phases (Fig. 1A), where M stands for the transition metal (e.g., Ti, Nb, Mo, V, W, etc.) and X stands for $\mathrm{C}$ or N, by selectively etching the main group element $\mathrm{A}$ (e.g., $\mathrm{Al}, \mathrm{Ga}, \mathrm{Si}$, etc.). The etching is usually performed in aqueous HF solutions rendering MXenes terminated with a mixture of $\mathrm{F}, \mathrm{O}$, and $\mathrm{OH}$ functional groups, commonly denoted as $\mathrm{T}_{x}$. These functional groups can be chemically modified, unlike the surfaces of other 2D materials such as graphene and transitionmetal dichalcogenides. Recent theoretical studies predict that selective terminations of MXenes with different surface groups can lead to remarkable properties, such as opening or closing bandgap (8), room-temperature electron mobility exceeding $10^{4} \mathrm{~cm}^{2} / \mathrm{V} \cdot \mathrm{s}(9)$, widely tunable work functions (10), half-metallicity, and 2D ferromagnetism (11). Covalent functionalization of MXene surfaces is expected to uncover new directions for rational engineering of $2 \mathrm{D}$ functional materials.

The surface of MXene sheets is defined during MAX phase etching. Electrochemical and hydrothermal methods have been recently applied for etching MAX phases without resorting to HF, but the use of aqueous solutions introduces a mixture of $\mathrm{Cl}, \mathrm{O}$, and $\mathrm{OH}$ surface groups $(12,13)$. The etching of $\mathrm{Ti}_{3} \mathrm{AlC}_{2}$ MAX phase in molten $\mathrm{ZnCl}_{2}$ and several other Lewis acidic molten salts above $500^{\circ} \mathrm{C}$ results in $\mathrm{Ti}_{3} \mathrm{C}_{2} \mathrm{Cl}_{2}$ MXene with a pure $\mathrm{Cl}$ termination $(14,15)$. Because etching of MAX phases in molten salts eliminates undesired oxidation and hydrolysis, we used a variation of this method for synthesis of $\mathrm{Ti}_{3} \mathrm{C}_{2} \mathrm{Cl}_{2}, \mathrm{Ti}_{2} \mathrm{CCl}_{2}$, and $\mathrm{Nb}_{2} \mathrm{CCl}_{2}$ MXenes in $\mathrm{CdCl}_{2}$ molten salt (figs. S1 to S5). Moreover, the use of Lewis acidic $\mathrm{CdBr}_{2}$ allowed us to extend the molten salt etching route beyond chlorides to prepare the first $\mathrm{Br}$-terminated $\mathrm{Ti}_{3} \mathrm{C}_{2} \mathrm{Br}_{2}$ and $\mathrm{Ti}_{2} \mathrm{CBr}_{2}$ MXenes (Fig. 1, B and C, and figs. S6 and S7). The morphology, structure, and composition of all new MXenes were characterized using high-resolution scanning transmission electron microscopy (STEM), Raman spectroscopy, and a combination of x-ray methods, including energy-dispersive elemental mapping, diffraction (XRD), atomic pair distribution function (PDF), fluorescence (XRF), extended x-ray absorption fine structure (EXAFS), and photoelectron spectroscopy (XPS).

We show that Cl- and, especially, Br-terminated MXenes can efficiently engage in a new type of surface reactions where halide ions exchange for other atoms and functional groups. It enables unprecedented control over the surface chemistry, structure, and properties of MXene materials.

The transition-metal atoms from the outer layers of MXene sheets ( $\mathrm{Ti}, \mathrm{Mo}, \mathrm{Nb}, \mathrm{V}$ ) form relatively weak $\mathrm{M}-\mathrm{Cl}$ and $\mathrm{M}-\mathrm{Br}$ bonds, in comparison to M-F and M-OH bonds typical for MXenes with $\mathrm{T}_{x}$ surface groups. This point can be demonstrated by the enthalpies of formation for $\operatorname{TiBr}_{4}(-617 \mathrm{~kJ}$ $\left.\mathrm{mol}^{-1}\right)$ and $\mathrm{TiCl}_{4}\left(-804 \mathrm{~kJ} \mathrm{~mol}^{-1}\right)$ versus $\mathrm{TiF}_{4}\left(-1649 \mathrm{~kJ} \mathrm{~mol}^{-1}\right)$, as well as by direct comparison of the bond energies (table S1). Strong Ti-F and Ti-O bonds make it difficult to perform any postsynthetic covalent surface modifications of MXenes (16). In contrast, Cl- and Br-terminated MXenes with labile surface bonding act as versatile synthons for further chemical transformations. 
MXene surface exchange reactions typically require temperatures of $300^{\circ}$ to $600^{\circ} \mathrm{C}$, which are difficult to achieve using traditional solvents. Instead, we used molten alkali metal halides as solvents with unmatched high-temperature stability, high solubility of various ionic compounds, and wide electrochemical windows (17-19). For example, $\mathrm{Ti}_{3} \mathrm{C}_{2} \mathrm{Br}_{2}$ MXene (Fig. 1B) dispersed in $\mathrm{CsBr} / \mathrm{KBr} / \mathrm{LiBr}$ eutectic (m.p. $236^{\circ} \mathrm{C}$ ) reacted with $\mathrm{Li}_{2} \mathrm{Te}$ and $\mathrm{Li}_{2} \mathrm{~S}$ to form new $\mathrm{Ti}_{3} \mathrm{C}_{2} \mathrm{Te}$ (Fig. $1 \mathrm{D}$ and figs. $\mathrm{S} 8$ to S10) and $\mathrm{Ti}_{3} \mathrm{C}_{2} \mathrm{~S}$ (Fig. $1 \mathrm{E}$ and fig. S11) MXenes, respectively. The reactions of $\mathrm{Ti}_{3} \mathrm{C}_{2} \mathrm{Cl}_{2}$ and $\mathrm{Ti}_{3} \mathrm{C}_{2} \mathrm{Br}_{2}$ with $\mathrm{Li}_{2} \mathrm{Se}$, $\mathrm{Li}_{2} \mathrm{O}$, and $\mathrm{NaNH}_{2}$ yielded $\mathrm{Ti}_{3} \mathrm{C}_{2} \mathrm{Se}, \mathrm{Ti}_{3} \mathrm{C}_{2} \mathrm{O}$, and $\mathrm{Ti}_{3} \mathrm{C}_{2}(\mathrm{NH})$ MXenes, respectively (figs. S12 to S16). The multilayers of $\mathrm{Ti}_{3} \mathrm{C}_{2} \mathrm{~T}_{n}$ MXenes $(\mathrm{T}=\mathrm{Cl}, \mathrm{S}, \mathrm{NH})$ were further treated with $n$ butyl lithium resulting in $\mathrm{Li}^{+}$intercalated sheets (fig. S17) with a negative surface charge (Fig. 2A and fig. S18). Subsequent dispersion in a polar organic solvent such as $N$-methyl formamide (NMF) resulted in stable colloidal solutions of single-layer flakes (Fig. 2, B and C). Raman spectroscopy and elemental analysis show that delaminated MXenes preserve their original $\mathrm{T}_{n}$ surface groups (figs. S18 to S20). X-ray diffraction pattern of spin-coated films showed a single (0002) diffraction peak corresponding to the center-to-center separation ( $d$ ) between two adjacent MXene sheets (Fig. 2D). The absence of $(10 \overline{1} l)$ and $(11 \overline{2} 0)$ reflections is consistent with the alignment of delaminated flakes parallel to the substrate (20), which is also confirmed by the grazing incidence wide angle X-ray scattering patterns (fig. S19).

Similar covalent surface modifications were achieved for $\mathrm{Ti}_{2} \mathrm{CCl}_{2}, \mathrm{Ti}_{2} \mathrm{CBr}_{2}$, and $\mathrm{Nb}_{2} \mathrm{CCl}_{2} \mathrm{MXenes} \mathrm{(Fig.} \mathrm{3A} \mathrm{and} \mathrm{figs.} \mathrm{S21} \mathrm{to}$ S34). The ability to perform surface exchange reactions on the thinnest MXenes demonstrated that the 2D sheets remained intact during all stages of the transformation. The exact metal/surface group elemental ratios for new MXenes were near the expected values as summarized in table S2.

The reactions of $\mathrm{Ti}_{3} \mathrm{C}_{2} \mathrm{Br}_{2}$ and $\mathrm{Ti}_{2} \mathrm{CBr}_{2}$ with $\mathrm{LiH}$ at $300^{\circ} \mathrm{C}$ produced bare $\mathrm{Ti}_{3} \mathrm{C}_{2} \square_{2}$ (Fig. $1 \mathrm{~F}$ and fig. S14) and $\mathrm{Ti}_{2} \mathrm{C}_{2}$ MXenes (fig. S21), where $\square$ stands for the vacancy site. Because $\mathrm{H}$-terminations are difficult to observe by STEM and other methods, we based this conclusion on the experimental value of the center-to-center distance between the $\mathrm{Ti}_{3} \mathrm{C}_{2}$ sheets (7.59 $\AA$ ), which was substantially smaller than the theoretical prediction for $\mathrm{Ti}_{3} \mathrm{C}_{2} \mathrm{H}_{2}$ MXene (8.26 $\AA$ ) and near the smallest theoretically possible spacing of $7.23 \AA$ (21). Because XPS showed reduction of Ti (fig. S16), this process could be formally described as a reductive-elimination of the hydride groups following the exchange reaction.

The chemical transformations of solids are generally impeded by slow diffusion, which severely limits the scope of synthesizable solid-state compounds (22). The complete exchange of surface groups in stacked MXenes is also expected to be kinetically cumbersome, especially if the entering ions are bulkier than the leaving ones, as in the case of $\mathrm{Cl}^{-}$(the ionic radius, $\left.R_{\mathrm{i}}=1.81 \AA\right)$ exchanged for $\mathrm{Te}^{2-}\left(R_{\mathrm{i}}=2.21 \AA\right)$. Counterintuitively, the reactions of $\mathrm{Ti}_{3} \mathrm{C}_{2} \mathrm{Cl}_{2}$ and $\mathrm{Ti}_{2} \mathrm{CCl}_{2}$ MXenes with $\mathrm{O}^{2-}, \mathrm{S}^{2-}, \mathrm{Se}^{2-}$, and $\mathrm{Te}^{2-}$ occurred at similar temperatures and with comparable reaction rates.

To understand this reactivity, we followed the evolution of the (0002) diffraction peak during surface exchange reactions. In the initial state, $\mathrm{Ti}_{3} \mathrm{C}_{2} \mathrm{Cl}_{2}$ sheets formed stacks (fig. S1) with $d=11.25 \AA$, and the van der Waals (vdW) gap between MXenes is $\sim 2.8 \AA$ (table S3), which is smaller than the dimensions of entering or leaving ions. No measurable changes of the $d$-spacing were detected upon heating $\mathrm{Ti}_{3} \mathrm{C}_{2} \mathrm{Cl}_{2}$ in $\mathrm{KCl}-\mathrm{LiCl}$ molten salt to $500^{\circ} \mathrm{C}$ (fig. S35). However, heating MXene in the same molten salt but in the presence of $\mathrm{Li}_{2} \mathrm{O}$ resulted in $d=13.2 \AA$ (fig. S36), which corresponds to a 4.7 to $6.3 \AA \mathrm{vdW}$ gap between the surface atoms on adjacent MXene sheets, depending on the local surface terminations (see supplementary materials). A similar $d=13.5 \AA$ was observed during reaction of $\mathrm{Ti}_{3} \mathrm{C}_{2} \mathrm{Cl}_{2}$ MXene with $\mathrm{Li}_{2} \mathrm{Se}$, although with a larger disorder (fig. S37).

The unstacking of MXene sheets in molten salts greatly facilitated diffusion of ions and made MXene surfaces sterically accessible. The interaction potential of MXenes in a molten salt was likely defined by surface-templated ion layering which created an exponentially decaying oscillatory interaction energy (23). We speculate that the free energy released in the surface exchange reaction caused MXene sheets to "swell" into one of the energy minima and stay in this state during chemical transformation.

Moreover, the nature of the surface groups had an unusually strong impact on the MXene structure. The XRD patterns of $\mathrm{Ti}_{3} \mathrm{C}_{2} \mathrm{~T}_{n}$ and majority of $\mathrm{Ti}_{2} \mathrm{CT}_{n}$ MXenes were modeled using the space group of the parent $\mathrm{Ti}_{3} \mathrm{AlC}_{2}$ and $\mathrm{Ti}_{2} \mathrm{AlC}$ MAX phases $\left(P 6_{3} / m m c\right)(24)$. Due to the simpler structure of thinner $\mathrm{Ti}_{2} \mathrm{CT}_{n}$ MXenes, their representative XRD patterns were further modeled using the Rietveld refinement. The fitting of the experimental Fourier-transformed EXAFS functions of $\mathrm{Ti}_{2} \mathrm{CT}_{n}$ MXenes (fig. S26) demonstrated that the local structure around Ti atoms was consistent with the respective crystallographic models. The real space interatomic PDFs, G(r), showed systematic shifts of Ti-T and Ti-Ti2 distances to larger values in $\mathrm{S}$ to Te series of $\mathrm{Ti}_{2} \mathrm{CT}_{n}$ MXenes (Fig. 3, A and $\mathrm{B}$, and fig. S27). In MXenes, Ti-Ti2 distance is equal to the nearestneighbor distance between Ti atoms in the basal (0001) plane and hence it represents the in-plane $a$ lattice constant (Fig. 3, $\mathrm{B}$ and C). For example, for $\mathrm{Ti}_{2} \mathrm{CBr}_{2}$ the Rietveld, EXAFS, and PDF methods converged on $a=3.32 \AA$. After exchanging $\mathrm{Br}^{-}$ for $\mathrm{O}^{2-}$, the resultant MXene showed $a=3.01 \AA$, and the reaction with $\mathrm{Te}^{2-}$ produced MXene with $a=3.62 \AA$ (Fig. 3C). The simulated XRD patterns of $\mathrm{Ti}_{2} \mathrm{CT}_{n}$ MXenes (figs. S38 and S39) suggest that large $\mathrm{Te}^{2-}$ groups are likely positioned on top of the neighboring Ti atoms (Fig. 3D). This is distinctively 
different from the MXenes with smaller surface groups which are positioned between hexagonally packed Ti surface atoms, on top of the opposite Ti atoms of the same $\mathrm{Ti}_{2} \mathrm{CT}_{n}$ sheet (Fig. $3 \mathrm{~B}$ ), in accordance with recent theoretical studies (25).

The vdW radii and packing density of surface atoms had a huge effect on $a$ (Fig. 3C), and fig. S40 compares these values with available computational predictions. For comparable ion radii, e.g., $\mathrm{S}$ versus $\mathrm{Cl}$ and $\mathrm{Se}$ versus $\mathrm{Br}$, halidoterminated MXenes showed larger $a$, likely because of the smaller number of chalcogenide ions required for charge compensation of the MXene surface. To estimate the in-plane strain $\left(\varepsilon_{\|}\right)$imposed on the titanium carbide lattice by surface groups in the new MXene species, we compared $a$ to the nearest-neighbor distance between Ti atoms in (111) plane of bulk cubic TiC that is structurally equivalent to the basal (0001) MXene plane. For $\mathrm{Ti}_{3} \mathrm{C}_{2} \mathrm{~T}_{n}$ and $\mathrm{Ti}_{2} \mathrm{CT}_{n}$ MXene families, the mixed $\left(\mathrm{T}_{x}=\mathrm{F}, \mathrm{O}, \mathrm{OH}\right)$ and pure $\mathrm{O}^{2-}$ terminations resulted in a compressive $\varepsilon_{\|}$. Bare ( $\square$ ) and NH-terminated MXenes were nearly strain-free, whereas $\mathrm{Cl}, \mathrm{S}$, Se, and Br-terminated MXenes all had tensile $\varepsilon_{\|}$. The thinner $\mathrm{Ti}_{2} \mathrm{CT}_{n}$ MXenes had, on average, a slightly larger in-plane expansion or contraction with respect to the bulk TiC lattice, compared to the thicker $\mathrm{Ti}_{3} \mathrm{C}_{2} \mathrm{~T}_{n}$ MXenes. The $\mathrm{Ti}_{2} \mathrm{CTe}$ MXene (Fig. 3, A and C, and fig. S24) had the largest magnitude of tensile $\varepsilon_{\|}$of $18.2 \%$ in accordance with $\mathrm{Te}^{2-}$ having the largest $\mathrm{vdW}$ radius among all groups used in this study. This degree of lattice expansion in a crystalline solid is very unusual. For comparison, the lattice of bulk TiC expands by "only" $2.5 \%$ when heated from room temperature to $2700^{\circ} \mathrm{C}(26)$.

Since the out-of-plane $c$ lattice constant is strongly affected by the intercalation of ions and solvent molecules between MXene sheets (27), we used high-resolution STEM images to assess the distances between the Ti planes along the $c$ axis of the unit cell (table S3). The magnitude of the outof-plane strain in the MXene core $\left(\varepsilon_{\perp}\right)$ was calculated by referencing experimental distances between Ti planes inside the MXene sheets $\left(M_{\perp}\right)$ to the distance between the (111) planes of bulk TiC (table S3). Figure 3E shows that the expansion of the $a$-lattice parameter in $\mathrm{Ti}_{3} \mathrm{C}_{2} \mathrm{~T}_{n}$ MXenes functionalized with $\mathrm{S}, \mathrm{Cl}, \mathrm{Se}, \mathrm{Br}$, and Te atoms was accompanied by the corresponding contraction of the $\mathrm{Ti}_{3} \mathrm{C}_{2}$ layers along the $c$ axis.

This observation is consistent with the behavior of the $\mathrm{Ti}_{3} \mathrm{C}_{2}$ layers as an elastic 2D sheet under tensile stress imposed by the surface atoms (Fig. 3E). The Poisson effect can account for the relations between the stress and the strain components reflected by observed changes of $a$ and $M_{\perp}$ distances. Unfortunately, our atomic-resolution STEM images of MXenes measured $M_{\perp}$ values with relatively large error bars (caused by projection effects and bending of the MXene sheets), which complicated an accurate estimation of the Poisson's ratio $(v)$ for new MXenes. A simple elastic model (see supplementary materials) applied to $\mathrm{Ti}_{3} \mathrm{C}_{2} \mathrm{~T}_{n}$ yields $v \sim 0.22$ for $\mathrm{T}=\mathrm{S}$ and $\mathrm{Br}$, which is comparable to the recently predicted $v$ value for $\mathrm{Ti}_{3} \mathrm{C}_{2} \mathrm{~T}_{x}(21)$. However, $\mathrm{Ti}_{3} \mathrm{C}_{2} \mathrm{Te}$ showed $v=0.16 \pm 0.06$, likely caused by the additional stiffening of the $\mathrm{Ti}_{3} \mathrm{C}_{2}$ layers under very large in-plane stress.

The above examples show that the composition and structure of MXenes can be engineered with previously unattainable versatility. Chemical functionalization of MXene surfaces is expected to impact nearly every property of these materials, and we found that the surface groups defined the nature of electronic transport in $\mathrm{Nb}_{2} \mathrm{CT}_{n}$ MXenes. Figure 4, A and $\mathrm{B}$, shows temperature-dependent four-probe resistivity ( $\rho$ ) measured on cold-pressed pellets of $\mathrm{Nb}_{2} \mathrm{CT}_{n}(\mathrm{~T}=\square, \mathrm{Cl}, \mathrm{O}$, $\mathrm{S}, \mathrm{Se}) \mathrm{MXenes}$ (fig. S41), all synthesized by the procedures described above. Figure 4A also compares the conductivity of the parent $\mathrm{Nb}_{2} \mathrm{AlC}$ MAX phase with that of $\mathrm{Nb}_{2} \mathrm{CCl}_{2}$ MXene. Above $30 \mathrm{~K}$, both MAX phase and MXene samples showed similar specific resistivity, which decreased when the sample is cooled. This temperature dependence is often associated with metallic conductivity. The ultraviolet photoelectron spectroscopy (UPS) confirmed nonzero density of electronic states at the Fermi energy $E_{\mathrm{F}}$ (fig. S42), which is also consistent with a metallic state.

However, when the $\mathrm{Nb}_{2} \mathrm{CCl}_{2}$ MXene was cooled below 30 $\mathrm{K}$, the resistivity started increasing, possibly indicating the onset of localization. A sharp drop of resistivity by several orders of magnitude occurred at a critical temperature $T_{\mathrm{c}} \sim 6.0$ $\mathrm{K}$ (Fig. 4A), which is reminiscent of a superconductive transition. The magnetic susceptibility measurements showed the development of a strong diamagnetism below $6.3 \mathrm{~K}$ that we interpreted as the Meissner effect (Fig. 4A). From the magnitude of zero-field-cooled data at $1.8 \mathrm{~K}$, we estimated the lower bound for the superconducting volume fraction of $\mathrm{Nb}_{2} \mathrm{CCl}_{2}$ MXene as $\sim 35 \%$. Consistent with superconductivity, the transition broadened, and $T_{\mathrm{c}}$ shifted to lower temperatures with the application of external magnetic field (Fig. 4B and fig. $\mathrm{S} 43$ ). In contrast, the parent $\mathrm{Nb}_{2} \mathrm{AlC}$ MAX phase exhibited normal metal behavior down to the lowest measured temperature $(1.8 \mathrm{~K})$, which is consistent with $T_{\mathrm{c}} \sim 0.44 \mathrm{~K}$ previously reported for $\mathrm{Nb}_{2} \mathrm{AlC}$ (28). For reference, $\mathrm{Nb}_{2} \mathrm{CT}_{x}$ MXene with mixed $\mathrm{O}, \mathrm{OH}, \mathrm{F}$ termination prepared by the traditional aqueous HF etching route shows two orders of magnitude higher resistivity and no superconductivity (fig. S44) (29).

In contrast to $\mathrm{Nb}_{2} \mathrm{CCl}_{2}$ MXene, the resistivity of MXenes terminated with chalcogenide ions $(\mathrm{O}, \mathrm{S}, \mathrm{Se})$ gradually increased when the sample was cooled (Fig. 4B), consistent with the activated transport regime. Given that UPS showed the finite density of states at $E_{\mathrm{F}}$ in $\mathrm{Nb}_{2} \mathrm{CS}_{2}$ (fig. S42), we hypothesized that the localization was controlled by the tunneling rates for charge carriers between metallic MXene sheets. The oxo-terminated $\mathrm{Nb}_{2} \mathrm{CT}_{n}$ MXene showed the highest resistivity, and the seleno-terminated MXene the lowest resistivity, consistent with the reduction of the tunneling barrier 
heights between the MXene sheets.

In the low-temperature region, we observed superconducting transitions in $\mathrm{Nb}_{2} \mathrm{CS}_{2}\left(T_{\mathrm{c}} \sim 6.4 \mathrm{~K}\right), \mathrm{Nb}_{2} \mathrm{CSe}\left(T_{\mathrm{c}} \sim 4.5\right.$ $\mathrm{K})$, and $\mathrm{Nb}_{2} \mathrm{C}(\mathrm{NH})\left(T_{\mathrm{c}} \sim 7.1 \mathrm{~K}\right.$, fig. S34), whereas $\mathrm{Nb}_{2} \mathrm{CO}_{x}$ did not enter the superconducting state (fig. S45). In granular metals, the development of macroscopic superconductivity can be suppressed by weak coupling of individual superconducting domains, which is also reflected by the high resistivity in the normal state (30). The upper critical field $\left(\mu_{0} \mathrm{H}_{\mathrm{c} 2}\right)$ showed a strong dependence on the surface functional group. For example, $\mathrm{Nb}_{2} \mathrm{CS}_{2}$ MXene exhibited higher $\mu_{0} \mathrm{H}_{\mathrm{c} 2}$ compared to $\mathrm{Nb}_{2} \mathrm{CCl}_{2}$ (Fig. 4B, inset, and fig. S46). Interestingly, bare $\mathrm{Nb}_{2} \mathrm{C}_{\square_{2}}$ MXenes showed no transition to the superconducting state down to $1.8 \mathrm{~K}$ (Fig. 4B). Thus, surface groups were not spectators but active contributors to the MXene superconductivity, which is consistent with surface groups affecting biaxial lattice strain, phonon frequencies, and the strength of electron-phonon coupling.

The MXene exchange reactions represent an exciting counter example to traditional perception of solids as hard to post-synthetically modify entities. We showed that chemical bonds inside an extended MXene stack can be rationally designed in a way more typical for molecular compounds. Other MXene structures could be enabled by the combinations of etching and substitution reactions using Lewis acidic and Lewis basic molten salts, respectively.

\section{REFERENCES AND NOTES}

1. B. Anasori, M. R. Lukatskaya, Y. Gogotsi, 2D metal carbides and nitrides (MXenes) for energy storage. Nat. Rev. Mater. 2, 16098 (2017). doi:10.1038/natrevmats.2016.98

2. Y. Xia, T. S. Mathis, M.-Q. Zhao, B. Anasori, A. Dang, Z. Zhou, H. Cho, Y. Gogotsi, S. Yang, Thickness-independent capacitance of vertically aligned liquid-crystalline MXenes. Nature 557, 409-412 (2018). doi:10.1038/s41586-018-0109-z Medline

3. C. J. Zhang, S.-H. Park, A. Seral-Ascaso, S. Barwich, N. McEvoy, C. S. Boland, J. N. Coleman, Y. Gogotsi, V. Nicolosi, High capacity silicon anodes enabled by MXene viscous aqueous ink. Nat. Commun. 10, 849 (2019). doi:10.1038/s41467-01908383-y Medline

4. F. Shahzad, M. Alhabeb, C. B. Hatter, B. Anasori, S. Man Hong, C. M. Koo, Y. Gogotsi, Electromagnetic interference shielding with 2D transition metal carbides (MXenes). Science 353, 1137-1140 (2016). doi:10.1126/science.aag2421 Medline

5. V. Kamysbayev, N. M. James, A. S. Filatov, V. Srivastava, B. Anasori, H. M. Jaeger, Y. Gogotsi, D. V. Talapin, Colloidal gelation in liquid metals enables functional nanocomposites of 2D metal carbides (MXenes) and lightweight metals. ACS Nano 13, 12415-12424 (2019). doi:10.1021/acsnano.9b06207 Medline

6. J. Guo, B. Legum, B. Anasori, K. Wang, P. Lelyukh, Y. Gogotsi, C. A. Randall, Cold sintered ceramic nanocomposites of 2D MXene and zinc oxide. Adv. Mater. 30 , e1801846 (2018). doi:10.1002/adma.201801846 Medline

7. J. Zhang, Y. Zhao, X. Guo, C. Chen, C.-L. Dong, R.-S. Liu, C.-P. Han, Y. Li, Y. Gogotsi, G. Wang, Single platinum atoms immobilized on an MXene as an efficient catalyst for the hydrogen evolution reaction. Nat. Catal. 1, 985-992 (2018). doi:10.1038/s41929-018-0195-1

8. M. Khazaei, M. Arai, T. Sasaki, C.-Y. Chung, N. S. Venkataramanan, M. Estili, Y. Sakka, Y. Kawazoe, Novel electronic and magnetic properties of two-dimensional transition metal carbides and nitrides. Adv. Funct. Mater. 23, 2185-2192 (2013). doi:10.1002/adfm.201202502

9. L. Zhou, Y. Zhang, Z. Zhuo, A. J. Neukirch, S. Tretiak, Interlayer-decoupled Sc-based MXene with high carrier mobility and strong light-harvesting ability. J. Phys. Chem. Lett. 9, 6915-6920 (2018). doi:10.1021/acs.jpclett.8b03077 Medline
10. Y. Liu, H. Xiao, W. A. Goddard 3rd, Schottky-barrier-free contacts with twodimensional semiconductors by surface-engineered MXenes. J. Am. Chem. Soc. 138, 15853-15856 (2016). doi:10.1021/jacs.6b10834 Medline

11. C. Si, J. Zhou, Z. Sun, Half-metallic ferromagnetism and surface functionalizationinduced metal-insulator transition in graphene-like two-dimensional $\mathrm{Cr}_{2} \mathrm{C}$ crystals. ACS Appl. Mater. Interfaces 7, 17510-17515 (2015). doi:10.1021/acsami.5b05401 Medline

12. S.-Y. Pang, Y.-T. Wong, S. Yuan, Y. Liu, M.-K. Tsang, Z. Yang, H. Huang, W.-T. Wong, J. Hao, Universal strategy for HF-free facile and rapid synthesis of twodimensional MXenes as multifunctional energy materials. J. Am. Chem. Soc. 141, 9610-9616 (2019). doi:10.1021/jacs.9b02578 Medline

13. T. Li, L. Yao, Q. Liu, J. Gu, R. Luo, J. Li, X. Yan, W. Wang, P. Liu, B. Chen, W. Zhang, W. Abbas, R. Naz, D. Zhang, Fluorine-free synthesis of high-purity $\mathrm{Ti}_{3} \mathrm{C}_{2} \mathrm{~T}_{x}(\mathrm{~T}=\mathrm{OH}$, 0) via alkali treatment. Angew. Chem. Int. Ed. 57, 6115-6119 (2018). doj:10.1002/anie.201800887 Medline

14. M. Li, J. Lu, K. Luo, Y. Li, K. Chang, K. Chen, J. Zhou, J. Rosen, L. Hultman, P. Eklund, P. O. A. Persson, S. Du, Z. Chai, Z. Huang, Q. Huang, Element replacement approach by reaction with Lewis acidic molten salts to synthesize nanolaminated MAX phases and MXenes. J. Am. Chem. Soc. 141, 4730-4737 (2019). doi:10.1021/jacs.9b00574 Medline

15. Y. Li, H. Shao, Z. Lin, J. Lu, L. Liu, B. Duployer, P. O. A. Persson, P. Eklund, L. Hultman, M. Li, K. Chen, X. H. Zha, S. Du, P. Rozier, Z. Chai, E. Raymundo-Piñero, P. L. Taberna, P. Simon, Q. Huang, A general Lewis acidic etching route for preparing MXenes with enhanced electrochemical performance in non-aqueous electrolyte. Nat. Mater. (2020). Medline

16. D. Kim, T. Y. Ko, H. Kim, G. H. Lee, S. Cho, C. M. Koo, Nonpolar organic dispersion of $2 \mathrm{D} \mathrm{Ti}_{3} \mathrm{C}_{2} \mathrm{~T}_{x}$ MXene flakes via simultaneous interfacial chemical grafting and phase transfer method. ACS Nano 13, 13818-13828 (2019). doi:10.1021/acsnano.9b04088 Medline

17. V. Srivastava, V. Kamysbayev, L. Hong, E. Dunietz, R. F. Klie, D. V. Talapin, Colloidal chemistry in molten salts: Synthesis of luminescent $\ln _{1-x} G a_{x} P$ and $\ln _{1-x} G a_{x} A s$ quantum dots. J. Am. Chem. Soc. 140, 12144-12151 (2018). doi:10.1021/jacs.8b06971 Medline

18. A. Dash, R. Vaßen, O. Guillon, J. Gonzalez-Julian, Molten salt shielded synthesis of oxidation prone materials in air. Nat. Mater. 18, 465-470 (2019). doi:10.1038/s41563-019-0328-1 Medline

19. X. Liu, N. Fechler, M. Antonietti, Salt melt synthesis of ceramics, semiconductors and carbon nanostructures. Chem. Soc. Rev. 42, 8237-8265 (2013) doi:10.1039/C3CS60159E Medline

20. M. Ghidiu, M. W. Barsoum, The $\{110\}$ reflection in X-ray diffraction of MXene films: Misinterpretation and measurement via non-standard orientation. J. Am. Ceram. Soc. 100, 5395-5399 (2017). doi:10.1111/jace.15124

21. Z. H. Fu, Q. F. Zhang, D. Legut, C. Si, T. C. Germann, T. Lookman, S. Y. Du, J. S. Francisco, R. F. Zhang, Stabilization and strengthening effects of functional groups in two-dimensional titanium carbide. Phys. Rev. B 94, 104103 (2016) doi:10.1103/PhysRevB.94.104103

22. M. Jansen, A concept for synthesis planning in solid-state chemistry. Angew. Chem. Int. Ed. 41, 3746-3766 (2002). doi:10.1002/15213773(20021018)41:20<3746:AID-ANIE3746>3.0.C0:2-2 Medline

23. H. Zhang, K. Dasbiswas, N. B. Ludwig, G. Han, B. Lee, S. Vaikuntanathan, D. V. Talapin, Stable colloids in molten inorganic salts. Nature 542, 328-331 (2017). doi:10.1038/nature21041 Medline

24. M. Sokol, V. Natu, S. Kota, M. W. Barsoum, On the chemical diversity of the MAX phases. Trends Chem. 1, 210-223 (2019). doi:10.1016/j.trechm.2019.02.016

25. Y. Qin, X.-H. Zha, X. Bai, K. Luo, Q. Huang, Y. Wang, S. Du, Structural, mechanical and electronic properties of two-dimensional chlorine-terminated transition metal carbides and nitrides. J. Phys. Condens. Matter 32, 135302 (2019). doi:10.1088/1361-648X/ab6048 Medline

26. J. H. Richardson, Thermal expansion of three group IVA carbides to $2700^{\circ} \mathrm{C}$. J. Am. Ceram. Soc. 48, 497-499 (1965). doi:10.1111/i.1151-2916.1965.tb14647x

27. O. Mashtalir, M. Naguib, V. N. Mochalin, Y. Dall'Agnese, M. Heon, M. W. Barsoum, Y. Gogotsi, Intercalation and delamination of layered carbides and carbonitrides. Nat. Commun. 4, 1716 (2013). doi:10.1038/ncomms2664 Medline

28. T. H. Scabarozi, J. Roche, A. Rosenfeld, S. H. Lim, L. Salamanca-Riba, G. Yong, I. Takeuchi, M. W. Barsoum, J. D. Hettinger, S. E. Lofland, Synthesis and 
characterization of $\mathrm{Nb}_{2} \mathrm{AlC}$ thin films. Thin Solid Films 517, 2920-2923 (2009). doi:10.1016/i.tsf.2008.12.047

29. J. Halim, I. Persson, E. J. Moon, P. Kühne, V. Darakchieva, P. O. A. Persson, P. Eklund, J. Rosen, M. W. Barsoum, Electronic and optical characterization of 2D $\mathrm{Ti}_{2} \mathrm{C}$ and $\mathrm{Nb}_{2} \mathrm{C}$ (MXene) thin films. J. Phys. Condens. Matter 31, 165301 (2019). doi:10.1088/1361-648X/ab00a2 Medline

30. I. S. Beloborodov, A. V. Lopatin, V. M. Vinokur, K. B. Efetov, Granular electronic systems. Rev. Mod. Phys. 79, 469-518 (2007). doi:10.1103/RevModPhys.79.469

31. A. N. Beecher, X. Yang, J. H. Palmer, A. L. LaGrassa, P. Juhas, S. J. L. Billinge, J. S. Owen, Atomic structures and gram scale synthesis of three tetrahedral quantum dots. J. Am. Chem. Soc. 136, 10645-10653 (2014). doi:10.1021/ja503590h Medline

32. M. Alhabeb, K. Maleski, B. Anasori, P. Lelyukh, L. Clark, S. Sin, Y. Gogotsi, Guidelines for synthesis and processing of two-dimensional titanium carbide ( $\mathrm{Ti}_{3} \mathrm{C}_{2} \mathrm{~T}_{x}$ MXene). Chem. Mater. 29, 7633-7644 (2017). doi:10.1021/acs.chemmater.7b02847

33. M. Naguib, J. Halim, J. Lu, K. M. Cook, L. Hultman, Y. Gogotsi, M. W. Barsoum, New two-dimensional niobium and vanadium carbides as promising materials for $\mathrm{Li}$ ion batteries. J. Am. Chem. Soc. 135, 15966-15969 (2013). doi:10.1021/ja405735d Medline

34. P. Stephens, Phenomenological model of anisotropic peak broadening in powder diffraction. J. Appl. Cryst. 32, 281-289 (1999). doi:10.1107/S0021889898006001

35. E. B. Deeva, A. Kurlov, P. M. Abdala, D. Lebedev, S. M. Kim, C. P. Gordon, A. Tsoukalou, A. Fedorov, C. R. Müller, In situ XANES/XRD study of the structural stability of two-dimensional molybdenum carbide $\mathrm{Mo}_{2} \mathrm{CT}_{x}$ : Implications for the catalytic activity in the water-gas shift reaction. Chem. Mater. 31, 4505-4513 (2019). doi:10.1021/acs.chemmater.9b01105

36. B. H. Toby, R. B. Von Dreele, GSAS-II: The genesis of a modern open-source all purpose crystallography software package. J. Appl. Cryst. 46, 544-549 (2013). doi:10.1107/S0021889813003531

37. X. Qiu, J. W. Thompson, S. J. L. Billinge, PDFGetX2: A Gui-driven program to obtain the pair distribution function from X-ray powder diffraction data. J. Appl. Cryst. 37, 678 (2004). doi:10.1107/S0021889804011744

38. B. Ravel, M. Newville, ATHENA, ARTEMIS, HEPHAESTUS: Data analysis for X-ray absorption spectroscopy using IFEFFIT. J. Synchrotron Radiat. 12, 537-541 (2005). doi:10.1107/S0909049505012719 Medline

39. J. J. Rehr, R. C. Albers, Theoretical approaches to X-ray absorption fine structure. Rev. Mod. Phys. 72, 621-654 (2000). doi:10.1103/RevModPhys.72.621

40. J. Halim, M. R. Lukatskaya, K. M. Cook, J. Lu, C. R. Smith, L.-Å. Näslund, S. J. May, L. Hultman, Y. Gogotsi, P. Eklund, M. W. Barsoum, Transparent conductive twodimensional titanium carbide epitaxial thin films. Chem. Mater. 26, 2374-2381 (2014). doi:10.1021/cm500641a Medline

41. A. Sarycheva, Y. Gogotsi, Raman spectroscopy analysis of the structure and surface chemistry of $\mathrm{Ti}_{3} \mathrm{C}_{2} \mathrm{~T}_{x}$ MXene. Chem. Mater. 32, 3480-3488 (2020). doi:10.1021/acs.chemmater.0c00359

42. N. Zhang, Y. Hong, S. Yazdanparast, M. Asle Zaeem, Superior structural, elastic and electronic properties of 2D titanium nitride MXenes over carbide MXenes: A comprehensive first principles study. 2D Materials 5, 045004 (2018). doi:10.1088/2053-1583/aacfb3

43. Y.-R. Luo, Comprehensive Handbook of Chemical Bond Energies (CRC Press, 2007).

44. M. A. Hope, A. C. Forse, K. J. Griffith, M. R. Lukatskaya, M. Ghidiu, Y. Gogotsi, C. P. Grey, NMR reveals the surface functionalisation of $\mathrm{Ti}_{3} \mathrm{C}_{2} \mathrm{MXene}$. Phys. Chem. Chem. Phys. 18, 5099-5102 (2016). doi:10.1039/C6CP00330C Medline

45. J. Palisaitis, I. Persson, J. Halim, J. Rosen, P. O. A. Persson, On the structural stability of MXene and the role of transition metal adatoms. Nanoscale 10, 1085010855 (2018). doi:10.1039/C8NR01986J Medline

46. I. Persson, L.-A. Näslund, J. Halim, M. W. Barsoum, V. Darakchieva, J. Palisaitis, J. Rosen, P. O. A. Persson, On the organization and thermal behavior of functional groups on $\mathrm{Ti}_{3} \mathrm{C}_{2}$ MXene surfaces in vacuum. 2D Materials 5, 015002 (2017). doi:10.1088/2053-1583/aa89cd

47. S. I. Troyanov, E. M. Snigireva, V. B. Rybakov, X-ray diffraction study of phase transition in alfa-TiCl3. Zh. Neorg. Khim. 36, 1117-1122 (1991).
48. S. I. Troyanov, V. M. Ionov, V. B. Rybakov, Synthesis and crystal structures of

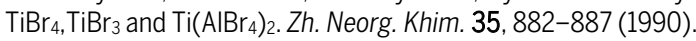

49. P. Ehrlich, W. Gutsche, H.-J. Seifert, Darstellung und kristallstruktur von titandibromid. Z. Anorg. Allg. Chem. 312, 80-86 (1961). doi:10.1002/zaac.19613120112

50. T. Hu, J. Wang, H. Zhang, Z. Li, M. Hu, X. Wang, Vibrational properties of $\mathrm{Ti}_{3} \mathrm{C}_{2}$ and $\mathrm{Ti}_{3} \mathrm{C}_{2} \mathrm{~T}_{2}(\mathrm{~T}=\mathrm{O}, \mathrm{F}, \mathrm{OH})$ monosheets by first-principles calculations: A comparative study. Phys. Chem. Chem. Phys. 17, 9997-10003 (2015). doi:10.1039/C4CP05666C Medline

51. N. C. Saha, H. G. Tompkins, Titanium nitride oxidation chemistry: An X-ray photoelectron spectroscopy study. J. Appl. Phys. 72, 3072-3079 (1992). doi:10.1063/1.351465

52. P. Ehrlich, Über titanselenide und -telluride. Zeitschrift für anorganische Chemie 260, 1-18 (1949). doi:10.1002/zaac.19492600102

53. M. Onoda, H. Wada, The titanium-sulphur system: Structures of $\mathrm{Ti}_{6.9} \mathrm{~S}_{9}(18 \mathrm{H})$ and $\mathrm{Ti}_{8.2} \mathrm{~S}_{11}(33 \mathrm{R})$ and the unit cells of $45 \mathrm{R}$ and $57 \mathrm{R}$ types of titanium sulphide. J. Less Common Met. 132, 195-207 (1987). doi:10.1016/0022-5088(87)90576-5

54. T. Hu, M. Hu, B. Gao, W. Li, X. Wang, Screening surface structure of MXenes by high-throughput computation and vibrational spectroscopic confirmation. J. Phys. Chem. C 122, 18501-18509 (2018). doi:10.1021/acs.jpcc.8b04427

55. J. Lu, I. Persson, H. Lind, J. Palisaitis, M. Li, Y. Li, K. Chen, J. Zhou, S. Du, Z. Chai, Z. Huang, L. Hultman, P. Eklund, J. Rosen, Q. Huang, P. O. A. Persson, $\mathrm{Ti}_{n+1} \mathrm{C}_{n}$ MXenes with fully saturated and thermally stable $\mathrm{Cl}$ terminations. Nanoscale $\mathrm{Adv}$. 1,3680-3685 (2019). doi:10.1039/C9NA00324J

56. G. R. Berdiyorov, Optical properties of functionalized $\mathrm{Ti}_{3} \mathrm{C}_{2} \mathrm{~T}_{2}(\mathrm{~T}=\mathrm{F}, \mathrm{O}, \mathrm{OH}) \mathrm{MXene}$ First-principles calculations. AIP Adv. 6, 055105 (2016). doi:10.1063/1.4948799

57. Q. Meng, J. Ma, Y. Zhang, Z. Li, C. Zhi, A. Hu, J. Fan, The S-functionalized $\mathrm{Ti}_{3} \mathrm{C}_{2}$ Mxene as a high capacity electrode material for $\mathrm{Na}$-ion batteries: A DFT study. Nanoscale 10, 3385-3392 (2018). doi:10.1039/C7NR07649E Medline

58. D. Wang, F. Li, R. Lian, J. Xu, D. Kan, Y. Liu, G. Chen, Y. Gogotsi, Y. Wei, A general atomic surface modification strategy for improving anchoring and electrocatalysis behavior of $\mathrm{Ti}_{3} \mathrm{C}_{2} \mathrm{~T}_{2} \mathrm{MXene}$ in lithium-sulfur batteries. ACS Nano 13, 11078-11086 (2019). doi:10.1021/acsnano.9b03412 Medline

59. R. Ibragimova, M. J. Puska, H.-P. Komsa, pH-dependent distribution of functional groups on titanium-based MXenes. ACS Nano 13, 9171-9181 (2019). doi:10.1021/acsnano.9b03511 Medline

\section{ACKNOWLEDGMENTS}

We thank Y. Gogotsi and B. Anasori (Drexel University) for kindly sharing the early samples of $\mathrm{Ti}_{3} \mathrm{AlC}_{2} \mathrm{MAX}$ phase and introducing us to an exciting field of MXenes. T. Witten, J. Anderson, J. Park, and B. Tian (UChicago) are acknowledged for many stimulating discussions. We also thank C. Malliakas (Northwestern) and A. Yakovenko (17-BM-B, APS) for transmission powder XRD, O. Borkiewicz (11-ID-B, APS) for X-ray total scattering, C.-J. Sun (20-BM-B, APS) for XAS, and J. Xie (UChicago) for magnetization measurements. Funding: V.K., H.H., D.W., and D.V.T. were supported by the Department of Defense (DOD) Air Force Office of Scientific Research under grant No. FA9550-18-1-0099; by the Office of Basic Energy Sciences, U.S. Department of Energy under Award No. DE-SC0019375; and by the National Science Foundation (NSF) under Award No. DMR-1611371. X.R., F.L., and R.F.K. were supported by the National Science Foundation (DMREF CBET-1729420). The JEOL JEM ARM200CF in the UIC Research Resources Center was acquired and upgraded using grants from the National Science Foundation (Grant No. DMR-0959470, DMR-1626065). This research used resources of the Center for Nanoscale Materials and the Advanced Photon Source, an Office of Science User Facilities operated for the U.S. Department of Energy (DOE) Office of Science by Argonne National Laboratory, supported by the U.S. DOE under Contract No. DE-AC02-06CH11357, and the Canadian Light Source and its funding partners. Author contributions: V.K. designed and performed the experiments, analyzed data, and co-wrote the paper. A.S.F. contributed to the X-ray data analysis. X.R., F.L., and R.F.K. performed high resolution STEM studies and image analysis. H.H. and D.W. carried out MAX phase synthesis and delamination of MXenes. D.V.T. conceived and designed experiments, analyzed data, co-wrote paper, and supervised the project. All authors discussed the results and commented on the manuscript. Competing interests: V.K. and D.V.T. are inventors on patent application U.S. 63/020,885 
submitted by the University of Chicago that covers surface modifications of MXenes and methods. Data and materials availability: All data needed to evaluate the conclusions in the paper are present in the paper or the supplementary materials.

\section{SUPPLEMENTARY MATERIALS}

science.sciencemag.org/cgi/content/full/science.aba8311/DC1

Materials and Methods

Supplementary Text

Figs. S1 to S46

Tables S1 to S15

References (31-59)

9 January 2020; accepted 2 June 2020

Published online 2 July 2020

10.1126/science.aba8311 


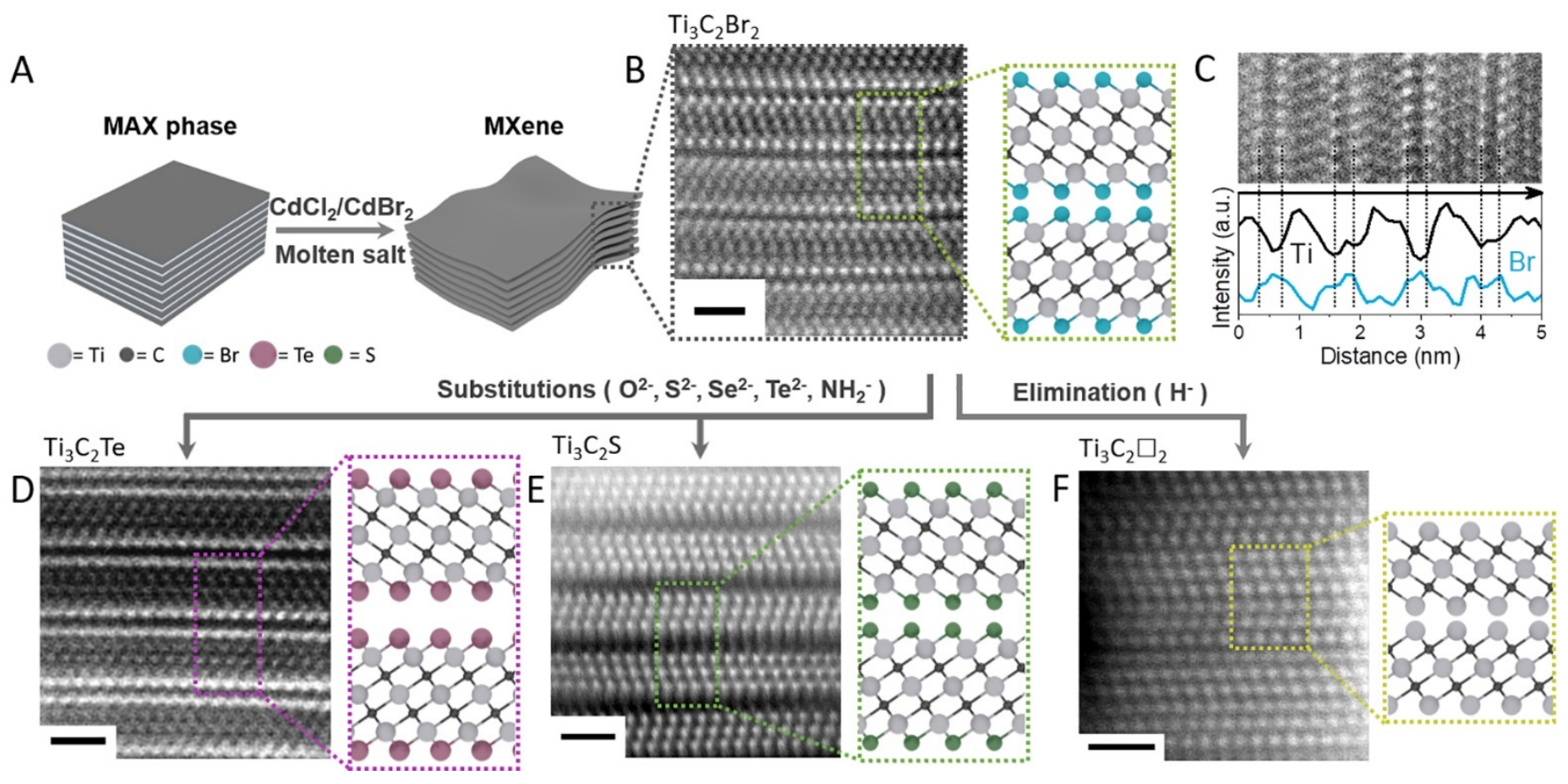

Fig. 1. Surface reactions of MXenes in molten inorganic salts. (A) Schematics for etching of MAX phases in Lewis acidic molten salts. (B) Atomic resolution high-angle annnular dark-field (HAADF) image of $\mathrm{Ti}_{3} \mathrm{C}_{2} \mathrm{Br}_{2}$ $M X e n e$ sheets synthesized by etching $\mathrm{Ti}_{3} \mathrm{AlC}_{2} \mathrm{MAX}$ phase in $\mathrm{CdBr}_{2}$ molten salt. The electron beam is parallel to $[2 \overline{1} \overline{1} 0]$ zone axis. (C) Energy dispersive $X$-ray (EDX) elemental analysis (line scan) of $\mathrm{Ti}_{3} \mathrm{C}_{2} \mathrm{Br}_{2} \mathrm{MX}$ ene sheets. HAADF images of (D) $\mathrm{Ti}_{3} \mathrm{C}_{2} \mathrm{Te}$ and $(\mathrm{E}) \mathrm{Ti}_{3} \mathrm{C}_{2} \mathrm{~S} M$ Menes obtained by substituting $\mathrm{Br}$ for $\mathrm{Te}$ and $\mathrm{S}$ surface groups, respectively. (F) HAADF image of $\mathrm{Ti}_{3} \mathrm{C}_{2} \square_{2}$ MXene ( $\square$ stands for the vacancy) obtained by reactive elimination of $\mathrm{Br}$ surface groups. All scale bars are $1 \mathrm{~nm}$. 


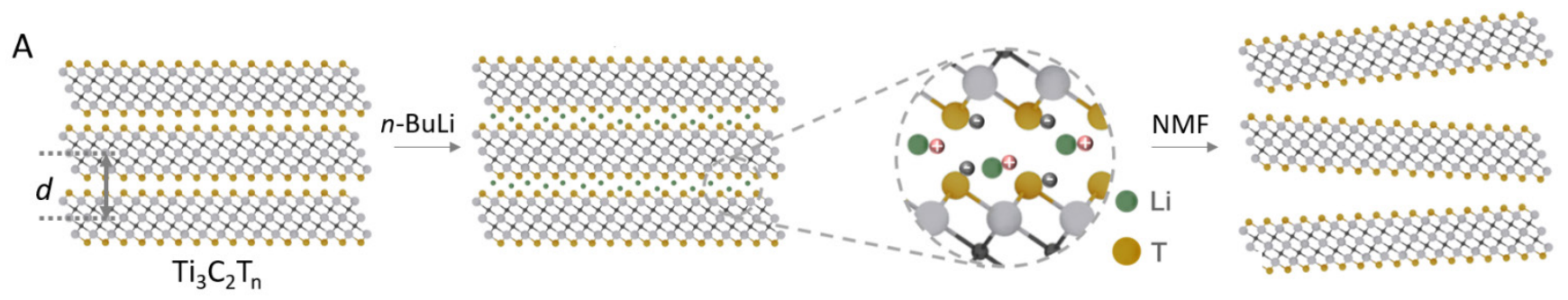

B
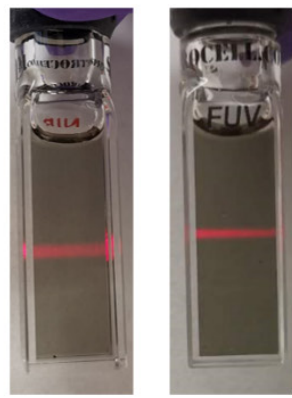

$\mathrm{Ti}_{3} \mathrm{C}_{2} \mathrm{Cl}_{2} \quad \mathrm{Ti}_{3} \mathrm{C}_{2} \mathrm{NH} \quad \mathrm{Ti}_{3} \mathrm{C}_{2} \mathrm{~S}$
$\mathrm{C}$

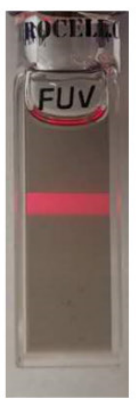

$\mathrm{C}$

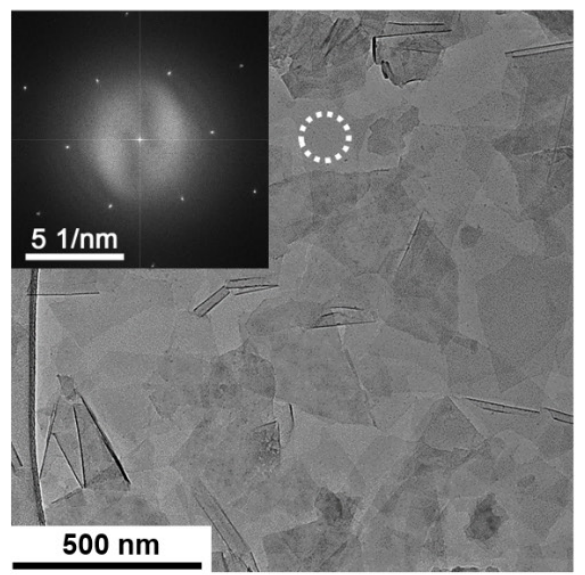

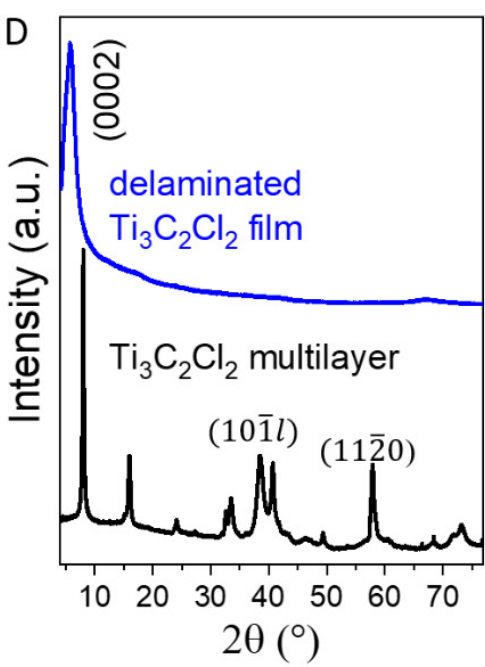

Fig. 2. Delamination of multilayer $\mathrm{Ti}_{3} \mathrm{C}_{2} \mathrm{~T}_{n}$ MXenes. (A) Schematic of delamination process. (B) Photographs of stable colloidal solutions of $\mathrm{Ti}_{3} \mathrm{C}_{2} \mathrm{~T}_{n}$ MXenes $(\mathrm{T}=\mathrm{Cl}, \mathrm{S}, \mathrm{NH})$ in NMF exhibiting Tyndall effect. (C) TEM image of $\mathrm{Ti}_{3} \mathrm{C}_{2} \mathrm{Cl}_{2}$ MXene flakes deposited from a colloidal solution. (Inset) Fast Fourier transform of the highlighted region showing crystallinity and hexagonal symmetry of the individual flake. (D) XRD patterns of multilayer MXene and delaminated flakes in a film spin casted on a glass substrate. 

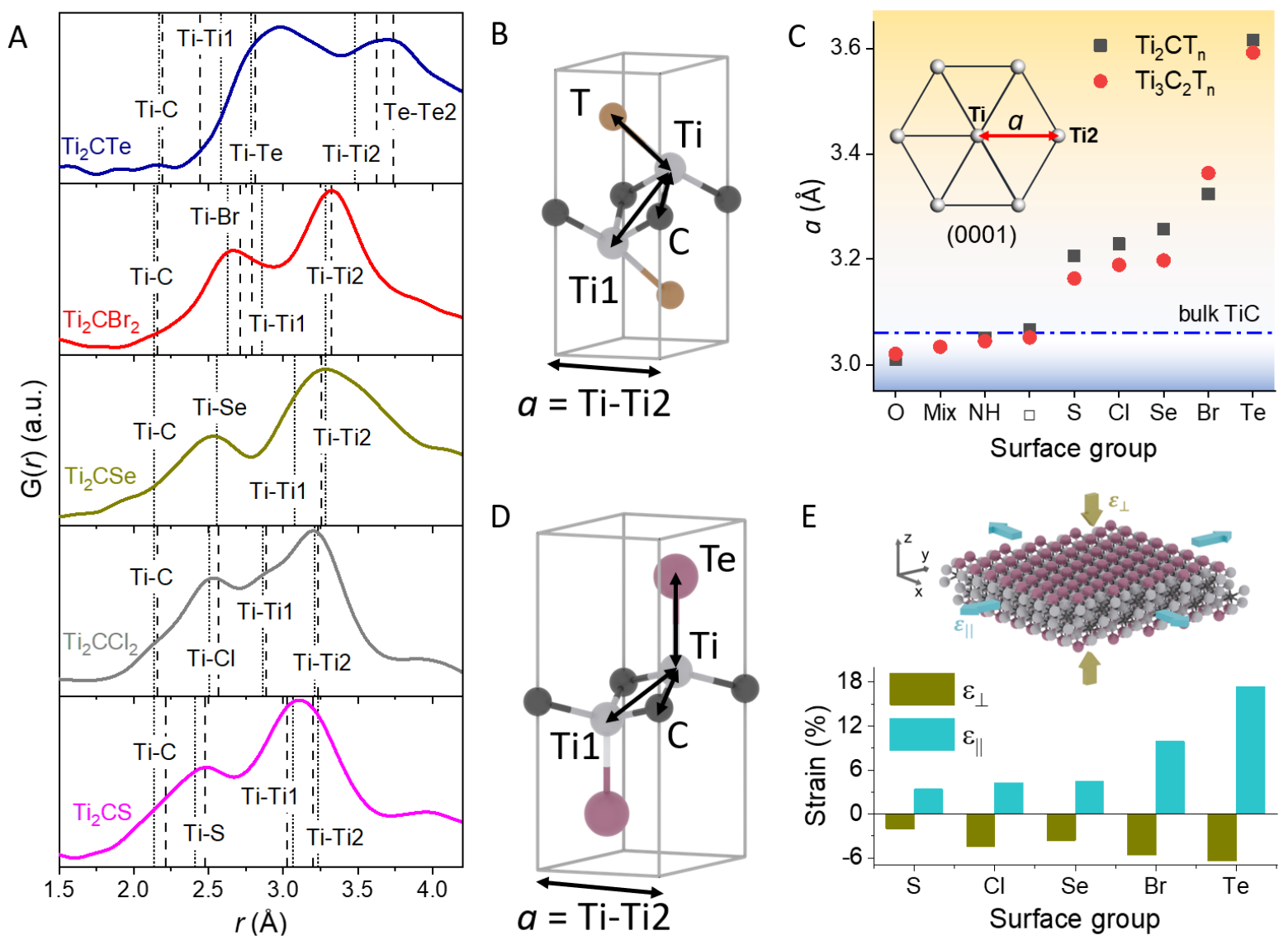

Fig. 3. Surface groups can induce giant strain in the MXene lattice. (A) Local interatomic distances in $\mathrm{Ti}_{2} \mathrm{CT}_{n}$ MXenes ( $\mathrm{T}=\mathrm{S}, \mathrm{Cl}$, Se, $\mathrm{Br}$ and $\mathrm{Te}$ ) probed by small $r$ region of the atomic pair distribution functions, $\mathrm{G}(r)$. The vertical lines show the Ti-C, Ti-T bond lengths and Ti-Ti1 and Ti$\mathrm{Ti} 2$ interatomic distances obtained from the Rietveld refinement of powder XRD patterns (dashed lines) and EXAFS analysis (dotted lines). (B) The unit cells of $\mathrm{Ti}_{2} \mathrm{CT}_{n}$ MXenes ( $\mathrm{T}=\mathrm{S}, \mathrm{Cl}, \mathrm{Se}, \mathrm{Br}$ ) obtained from the Rietveld refinement. (C) Dependence of the in-plane lattice constant a [equivalent to Ti-Ti2 distance in (A)] for $\mathrm{Ti}_{2} \mathrm{CT}_{n}$ and $\mathrm{Ti}_{3} \mathrm{C}_{2} \mathrm{~T}_{n} \mathrm{MXenes}$ on the chemical nature of the surface group $\left(T_{n}\right)$. (D) Proposed unit cell of $\mathrm{Ti}_{2} \mathrm{CTe}$ MXene (see fig. S39). (E) Biaxial straining of $\mathrm{Ti}_{3} \mathrm{C}_{2} \mathrm{~T}_{n}$ MXene lattice induced by the surface groups. The in-plane $\left(\varepsilon_{\|}\right)$and out-of-plane $\left(\varepsilon_{\perp}\right)$ strain components are evaluated with respect to the bulk cubic TiC lattice with $a_{\mathrm{TiC}}=4.32 \AA$. 

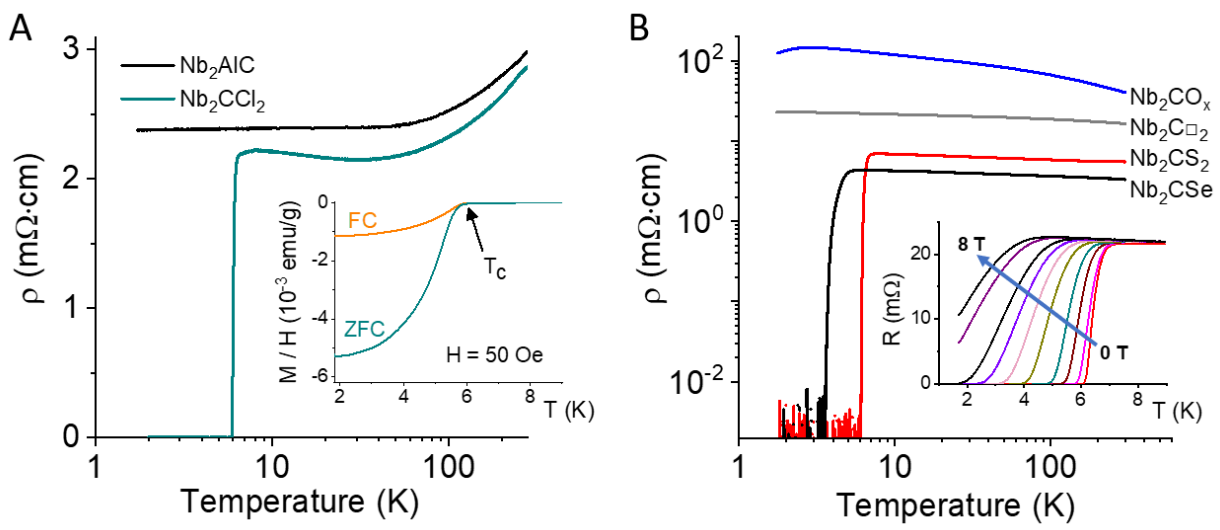

Fig. 4. Electronic transport and superconductivity in $\mathrm{Nb}_{2} \mathrm{CT}_{n}$ MXenes.

(A) Temperature-dependent resistivity for the cold-pressed pellets of $\mathrm{Nb}_{2} \mathrm{AIC}$ MAX phase and $\mathrm{Nb}_{2} \mathrm{CCl}_{2}$ MXene. (Inset) Magnetic susceptibility of $\mathrm{Nb}_{2} \mathrm{CCl}_{2} \mathrm{MXene}$ as a function of temperature. $\mathrm{FC}$ and ZFC correspond to the field cooled and zero-field cooled measurements, respectively. (B) Temperature-dependent resistivity for the cold pressed pellets of $\mathrm{Nb}_{2} \mathrm{CT}_{n}$ MXenes. (Inset) Resistance as a function of temperature at different applied magnetic fields ( 0 to $8 \mathrm{~T}$ ) for the cold-pressed pellets of $\mathrm{Nb}_{2} \mathrm{CS}_{2}$ MXene. 